Review Article

\title{
Anesthesia-Induced Oxidative Stress: Are There Differences between Intravenous and Inhaled Anesthetics?
}

\author{
Thomas Senoner (D), Corinna Velik-Salchner, Günter Luckner, and Helmuth Tauber
}

Dept. of Anesthesia and Critical Care Medicine, Medical University Innsbruck, Innsbruck, Austria

Correspondence should be addressed to Thomas Senoner; thomas.senoner@i-med.ac.at

Received 10 August 2021; Revised 11 October 2021; Accepted 12 November 2021; Published 27 November 2021

Academic Editor: Joël R. Drevet

Copyright (c) 2021 Thomas Senoner et al. This is an open access article distributed under the Creative Commons Attribution License, which permits unrestricted use, distribution, and reproduction in any medium, provided the original work is properly cited.

\begin{abstract}
Agents used for the induction of anesthesia have been shown to either promote or mitigate oxidative stress. A fine balance between the presence of reactive oxygen species (ROS) and antioxidants is crucial for the proper normal functioning of the cell. A basal concentration of ROS is essential for the manifestation of cellular functions, whereas disproportionate levels of ROS cause damage to cellular macromolecules such as DNA, lipids, and proteins, eventually leading to necrosis and apoptosis. Increased ROS has been linked with numerous illnesses, such as cardiovascular, immune system, liver, and kidney, and has been shown to promote cancer and accelerate aging. Knowledge of the various pharmacologic agents that increase or reduce oxidative stress may promote a safer way of inducing anesthesia. Furthermore, surgery itself leads to increased ROS production and ischemia/reperfusion injury. Indeed, increased perioperative oxidative stress has been correlated with increased postoperative complications and prolonged recovery. Anesthesiologists care for patients during the whole spectrum of perioperative care and thus are in a unique position to deliver countermeasures to oxidative stress. Using preferentially an induction agent which reduces oxidative stress might lead to better clinical outcomes and fewer postoperative complications. Propofol has been shown in several studies to reduce oxidative stress, which reduces postoperative complications and leads to a faster recovery, and thus might represent the preferred induction agent in the right clinical setting.
\end{abstract}

\section{Introduction}

Excess production of reactive oxygen species (ROS) has been implicated in the etiology of various chronic diseases such as neurodegenerative diseases, cardiovascular diseases, and cancer [1-4]. ROS comprise both oxygen free radicals, such as superoxide, hydroxyl radicals, and peroxyl radicals, and nonradicals, such as hydrogen peroxide, hypochlorous acid, and ozone [5]. In the majority of cell types, mitochondria are the main source of intracellular oxidant production, while nicotinamide adenine dinucleotide phosphate (NADPH) oxidases (summarized as the NOX family of enzymes) comprise other relevant drivers. Apart from that, numerous other enzymes such as xanthine oxidase, nitric oxide synthase, cyclooxygenases, cytochrome P450 enzymes, and lipoxygenases along with other cell organelles like the peroxisome and endoplasmic reticulum contribute to intracellular ROS production [6]. The main cellular structures afflicted by
ROS and reactive nitrogen species (RNS) are DNA, proteins, and lipids. ROS are constantly being generated and constitute a natural part of aerobic life. Indeed, basal levels of ROS are indispensable for the expression of numerous cellular functions, such as defense against invading microorganisms, signal transduction pathways, gene expression, and the promotion of growth or death [7]. Notwithstanding the central relevance of redox reactions, dysregulation of oxidant signaling may initiate or accelerate a host of pathological conditions, resulting in cellular dysfunction. However, the body is endowed with protective measures against ROS via enzymatic (e.g., superoxide dismutase (SOD), catalase (CAT), peroxiredoxin $(\operatorname{Prx})$, and glutathione peroxidase (GSH-Px)) as well as nonenzymatic compounds (e.g., tocopherol/vitamin E, beta-carotene, ascorbate, and glutathione (GSH)) [8].

The last few years have seen a rise in the incidence of surgical procedures being performed each year, which also translates to an increased number of people undergoing 
general or regional anesthesia [9]. Given this rise in anesthetic procedures being performed, delivering anesthesia in a safe manner becomes of utmost importance. Patients being cared for by anesthesiologists can be exposed to oxidative stress preoperatively, intraoperatively, and postoperatively [10]. Given the fact that the anesthesiologist cares for patients during all three of these phases, he or she has the potential to influence the extent to which the individual patient might be exposed to oxidative stress. However, perioperative oxidative stress is a complex response that comprises also patient and surgical factors. Nonetheless, anesthesiologists are in a unique position to convey countermeasures to oxidative stress and potentially improve the postoperative outcome. As such, a better understanding of the impact anesthetic agents play on oxidative stress and the clinical consequences is urgently needed so as to potentially improve patients' outcomes.

\section{Oxidative Stress and Postoperative Outcome}

The influence of preoperative risk factors and preoperative levels of oxidative stress is imperative in determining the degree of elevation of intraoperative oxidative stress. Additionally, the magnitude of the surgical intervention is a major determinant of intraoperative oxidative stress, with more invasive techniques being associated with increased oxidative stress levels compared with minimally invasive procedures [11]. Furthermore, oxidative stress has been shown to play an important role during different steps of many surgical procedures, such as transplantation (heart, liver, etc.), clamping/unclamping of the aorta (thoracic surgery and abdominal aortic surgery), intermittent inflow occlusion (hepatic and cardiac surgery), and release of a limb tourniquet (orthopedic surgery) [10]. Ischemia-reperfusion injury is a critical condition that often appears in more complex surgeries, with oxidative stress playing a major role in eliciting signaling pathways that lead to the onset of necrosis/apoptosis. During the reoxygenation phase, ROS inflict direct tissue damage and initiate a cascade of deleterious cellular responses which culminate in inflammation and cell death [10].

Preoperative levels of oxidative stress have been shown to predict an increased risk of delayed recovery and complications after surgery [12]. In 186 patients undergoing radical esophagectomy, the preoperative blood d-ROM (derivatives of reactive oxygen metabolite) level, as well as pre- and postoperative plasma FAC, was assessed to determine oxidative stress. Other markers such as white blood cell count, C-reactive protein level, incidence of severe postoperative complications, and postoperative recovery process within 30 days after surgery were also assessed in a double-blind fashion. In patients with elevated preoperative d-ROMs, postoperative levels of white blood cell count and C-reactive protein took longer to return to normal levels. Plasma FAC was decreased postoperatively, and the magnitude was positively correlated with preoperative d-ROM levels. The study further showed that patients receiving propofol had no postoperative decline in FAC, a decreased incidence of severe postoperative complications, and faster uneventful recovery time. An elevated preoperative d-ROM level is associated with increased intraoperative oxidative stress and more postoperative complications with prolonged recovery [12].

Another study analyzed the effect of coronary artery bypass grafting $(\mathrm{CABG})$ on oxidative stress and determined their associations with postoperative complications [13]. Asymmetric dimethylarginine (ADMA), which is associated with an increased risk of coronary artery disease, as well as 8-iso-prostaglandin $\mathrm{F}_{2 \alpha}$ (8-iso- $\mathrm{PGF}_{2 \alpha}$ ), a marker of increased lipid peroxidation, was measured preoperatively and twice postoperatively: $18-36 \mathrm{~h}$ and 5-7 days after surgery. The primary outcomes were in-hospital cardiovascular death and postoperative myocardial infarction.

ADMA increased by $68 \%$ at $18-36 \mathrm{~h}$ after CABG $(p=0.0001)$ and then decreased by $20 \%$ on postoperative days 5-7 $(p=0.0001)$. Similarly, 8 -iso- $\mathrm{PGF}_{2 \alpha}$ increased by $30 \%$ at $18-36 \mathrm{~h}$ post-CABG $(p=0.05)$ and then decreased by $11 \%$ on postoperative days $5-7(p<0.0001)$. There was a positive correlation between ADMA and 8-iso- $\mathrm{PGF}_{2 \alpha}$ at all time points $(r=0.53$ at baseline, $r=0.81$ at $18-36 \mathrm{~h}$, and $r=0.80$ on postoperative days $5-7, p<0.0001$ for all comparisons). Among the 158 patients, 13 (8.2\%) patients suffered a postoperative myocardial infarction. These patients had higher ADMA and 8-iso- PGF $_{2 \alpha}$ levels either at baseline or postoperatively as compared with patients who did not suffer a myocardial infarction. Six patients (3.8\%) died during the early postoperative period due to an extensive myocardial infarction. Also, these patients showed higher ADMA and 8-iso- GFF $_{2 \alpha}$ levels at all three time points compared with patients who survived. The alterations in the measured levels might, at least in part, be explained by enhanced ROS levels after surgery and show that increased oxidative stress is associated with the occurrence of adverse events after cardiac surgery [13].

Since postoperative atrial fibrillation (POAF) is associated with a longer hospital stay, an increased risk of future atrial fibrillation, heart failure, and overall mortality [14], Wu et al. [15] assessed the association of oxidative stress biomarkers ( $\mathrm{F}_{2}$-isoprostanes, isofurans, and $\mathrm{F}_{3}$-isoprostanes) in plasma and urine with incident POAF among 551 cardiac surgery patients. Biomarkers were measured at baseline, the end of surgery, and postoperative day 2. 171 patients developed POAF during follow-up. Oxidative stress biomarkers at baseline were not significantly associated with POAF. By the end of surgery, however, isofurans (both in urine and plasma) and $\mathrm{F}_{3}$-isoprostanes (in urine, but not plasma) were significantly associated with an elevated risk of POAF. $\mathrm{F}_{2}$-isoprostanes were not associated with a higher POAF risk. At postoperative day 2, plasma isofurans and $\mathrm{F}_{2}$-isoprostanes were both associated with an increased risk of POAF. Urine concentrations of these biomarkers as well as $\mathrm{F}_{3}$-isoprostane levels in both urine and plasma were not associated with subsequent POAF. There was overall a substantial association, with subject in the top quartile of oxidative stress biomarker having up to 3 -fold higher odds of POAF. The results of this study add to the growing evidence supporting the likely key pathogenic role of elevated oxidative stress in POAF [15].

Since evidence of increased oxidative stress and worse postoperative outcome is accumulating, the treating anesthesiologist 
might ask himself how her or she can reduce the increased oxidative stress that underlies all surgical interventions in order to potentially improve patients' outcomes. Given the many anesthetic agents at the hand of the anesthesiologist, analyzing the most commonly used anesthetic agents regarding their role in generating or mitigating oxidative stress seems reasonable. Thus, we will subsequently analyze first the most common inhaled anesthetics, which will be followed by a thorough analysis of the most commonly used intravenous anesthetics. Only human studies have been analyzed and included in this review.

\section{Inhaled Anesthetics}

Inhaled anesthetics are a chemically and pharmacologically distinct group that includes the potent halogenated ether (isoflurane, sevoflurane, desflurane, and enflurane) and alkane (halothane) volatile anesthetics and the inorganic gaseous anesthetics (nitrous oxide and xenon) [16]. These agents cause a composite pharmacological endpoint which is commonly being described as loss of consciousness, reversible amnesia, and immobility. These diverse effects result from multiple mechanisms which are unique for each individual agent, which vary in their relative potency and efficacy. Indeed, inhaled anesthetics have numerous, agentspecific effects on several molecular targets crucial to neuronal communication and excitability $[17,18]$. These various actions work synergistically or individually to bring about the pleiotropic effects innate to inhaled anesthetics [19].

We will subsequently analyze the most common inhaled anesthetics regarding their potential for inducing or mitigating oxidative stress.

Figure 1 represents the chemical structures of the inhaled anesthetics discussed in this paper.

Table 1 summarizes the inhaled anesthetic agents discussed in this paper regarding their effect on oxidative stress.

3.1. Sevoflurane. Sevoflurane is a fluorinated methyl isopropyl ether. It is well suited to inhalational inductions because it is minimally pungent and sweet smelling. Because it is completely fluorinated, sevofluranes' blood solubility is very low (among commonly used volatile anesthetics, only desflurane has a lower solubility), and it is about half as potent as isoflurane. Sevoflurane appears to induce the least cerebral vasodilation among the modern agents, and in fact, it has been shown that at 1 MAC (minimum alveolar concentration), cerebral autoregulation and $\mathrm{CO}_{2}$ reactivity are preserved, thus making sevoflurane the preferred volatile agent for neurosurgical patients with increased intracranial pressure $[20,21]$.

The impact of sevoflurane on the cognitive function and the expression of oxidative stress proteins among elderly patients undergoing radical surgery for lung cancer has been prospectively analyzed in a study conducted by Qin et al. [22] and compared with propofol administration. In both groups, serum S100 $\beta$ levels and expression of NOX2 and NOX4 proteins were determined in peripheral blood mononuclear cells. 24 hours after surgery, indicators of lung function such as FEV1 (forced expiration in the first second),
FVC (forced vital capacity), and VC (vital capacity) were higher in the sevoflurane group as compared to the propofol group $(p<0.001, p=0.008$, and $p=0.002$, respectively). At the end of the surgery and 24 hours after surgery, the Mini-Mental State Examination (MMSE) scores were higher in the sevoflurane group as compared to the propofol group (both $p<0.001$ ). $\mathrm{S} 100 \mathrm{~B}$, a calcium binding protein physiologically produced and released mainly by astrocytes, is considered a sensitive marker of central nervous system damage [23]. Both at the end and 24 hours after surgery, S100B levels were lower in the sevoflurane group ( $p=0.003$ and $p<0.001$, respectively). Furthermore, levels of markers of oxidative stress (NADPH oxidase subunit NOX2 and NOX4 proteins) in peripheral blood mononuclear cells were also found to be lower in the sevoflurane group $(p=0.033, p<0.001, p<0.001$, and $p<0.001$, respectively). The reduction in oxidative stress, as expressed by decreased levels of both NOX2 and NOX4, may mediate the improvement in lung function observed in this study [22].

Another study evaluated the DNA damage effects of sevoflurane and desflurane in human bronchoalveolar cells [24]. Patients undergoing lumbar discectomy surgery were recruited in the prospective study and randomized to either sevoflurane or desflurane for maintenance of anesthesia. Bronchoalveolar lavage samples and peripheral blood samples were collected at baseline $\left(T_{1}\right)$ and at the end of surgery $\left(T_{2}\right)$. Comet analysis was used to examine genotoxic properties. Plasma oxidative DNA damage was measured with 8 -hydroxy- $2^{\prime}$-deoxyguanosine ( 8 -OHdG), a commonly used biomarker for oxidative stress and carcinogenesis [25]. In both groups, comet parameters at baseline and at the end of surgery were significantly different, in that markedly higher values at $T_{2}$ were detected as opposed to baseline levels ( $p<0.05$ for desflurane and $p<0.05$ for sevoflurane). However, no statistically significant differences have been observed among the 2 groups in $T_{1}$ and $T_{2}$ comet parameter values $(p<0.05)$. In both the sevoflurane and desflurane groups, plasma 8-OHdG levels were increased at $T_{2}$ as compared with baseline levels $(p<0.05)$, while no significant difference emerged between the 2 groups at either time point $(p<0.05)$. In conclusion, both sevoflurane and desflurane may have genotoxic effects on bronchoalveolar cells. Furthermore, the authors observed elevated plasma levels of $8-\mathrm{OHdG}$, pointing toward increased oxidative damage to DNA [24].

There have been some indications in animal studies that sevoflurane might be neurotoxic in the developing brain, which is partly induced by increased oxidative stress [26-28]. A multicenter, randomized trial sought to investigate whether general anesthesia (GA) in infancy has any effect on the neurodevelopmental outcome compared with regional anesthesia (RA) [29]. In total, 532 infants younger than 60 weeks postmenstrual age who had inguinal herniorrhaphy were randomly assigned to receive either awake-regional anesthesia or sevoflurane-based general anesthesia. The main outcome assessed at 2 years of age was prespecified to be the composite cognitive score of the Bayley-III, which has cognitive, language, and motor scales. The median duration of anesthesia in the GA arm was 54 minutes. For the cognitive composite score, there was equivalence in means between arms (RA-GA: $+0.169,95 \% \mathrm{CI}-2.30$ to +2.64 ). Equivalence 

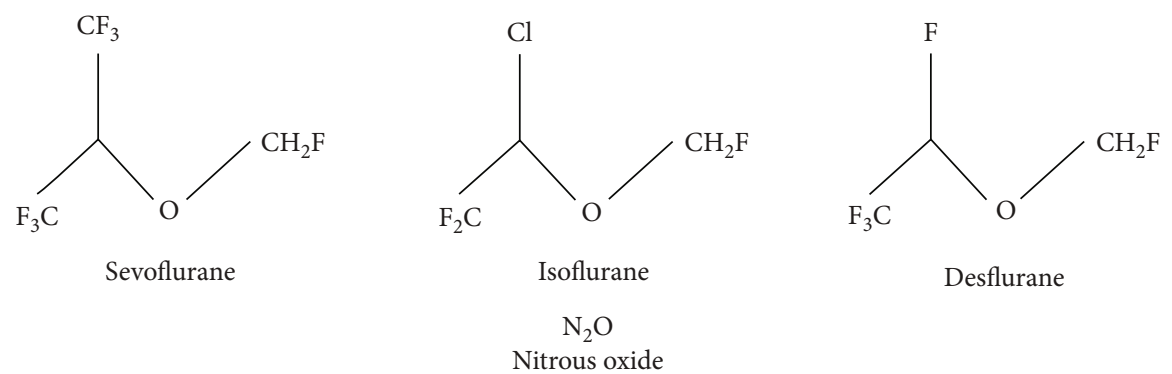

FIgURE 1: Chemical structures of the inhaled anesthetics discussed in this paper.

TABLE 1: Inhaled anesthetic agents investigated regarding their effect on oxidative stress.

\begin{tabular}{|c|c|c|c|}
\hline $\begin{array}{l}\text { Study } \\
\text { reference }\end{array}$ & Anesthetic agent & $\begin{array}{c}\text { Studied } \\
\text { population }\end{array}$ & Main effect on oxidative stress \\
\hline [22] & Sevoflurane & Human study & $\downarrow$ S100B levels, NOX2 and NOX4 protein levels compared with propofol treatment \\
\hline [24] & $\begin{array}{l}\text { Sevoflurane, } \\
\text { desflurane }\end{array}$ & Human study & $\begin{array}{l}\uparrow \text { Genotoxic effects on bronchoalveolar cells } \\
\uparrow \text { Plasma levels of } 8 \text {-OHdG }\end{array}$ \\
\hline [29] & Sevoflurane & Human study & Not $\uparrow$ risk of adverse neurodevelopmental outcome in infants \\
\hline [33] & Desflurane & Human study & $\begin{array}{c}\uparrow \% \text { of DNA strand breaks, } \uparrow \% \text { of oxidized pyrimidines, } \uparrow \text { levels of } 4 \text {-HNE and } \\
\text { 8-isoprostane, } \downarrow \% \text { of oxidized pyrimidines }\end{array}$ \\
\hline$[34]$ & Desflurane & Human study & $\uparrow$ LP values and $\downarrow$ vitamin E levels 1 hour following desflurane exposure \\
\hline [35] & $\begin{array}{l}\text { Sevoflurane, } \\
\text { desflurane }\end{array}$ & Human study & $\begin{array}{c}\text { Significantly greater } \uparrow \text { in postoperative LOOH, TOS, and OSI levels in desflurane-treated } \\
\text { pts compared with sevoflurane }\end{array}$ \\
\hline [43] & Isoflurane & Human study & $\uparrow$ DNA damage, inhibits repair of DNA damage by reducing p53 levels \\
\hline$[51]-[54]$ & Nitrous oxide & Human study & $\uparrow$ Sister chromatid exchange, chromosomal aberrations, DNA single-strand breaks \\
\hline
\end{tabular}

4-HNE: 4-hydroxynonenal; 8-OHdG: 8-hydroxy-2' -deoxyguanosine; DNA: deoxyribonucleic acid; LOOH: lipid hydroperoxide; LP: lipid peroxidation; NOX: NADPH oxidase; OSI: oxidative stress index; PTS: patients; TOS: total oxidant status.

was also achieved between arms in the composite motor scores, composite language scores, and composite adaptive behavior scores. In conclusion, this trial demonstrated that a less than one-hour exposure to a sevoflurane GA in infancy does not raise the risk of adverse neurodevelopmental outcome at two years of age [29].

3.2. Desflurane. Desflurane is a fluorinated methyl ethyl ether which is identical to isoflurane except for the substitution of a fluorine atom for the $\alpha$-ethyl chlorine. Its complete fluorination results in a markedly reduced blood solubility and potency, while vapor pressure relative to isoflurane is increased to almost atmospheric pressure. The low boiling point of desflurane necessitates a specialized pressurized vaporizer. Desflurane is the most pungent among the inhaled anesthetics and may cause coughing, sialorrhea, breath holding, and laryngospasm. Increased desflurane concentrations may lead to enhanced sympathetic activity with associated tachycardia and hypertension [30]. Because desflurane undergoes almost no metabolism, there is a very low risk of hepatitis and nephrotoxicity.

Some evidence suggests that desflurane may damage the DNA and depress the redox status $[31,32]$. A randomized clinical trial sought to investigate whether desflurane is associated with DNA damage and oxidative stress [33]. Blood samples were collected prior to premedication and anesthe- sia induction (baseline, $T_{1}$, one and a half hours after anesthesia induction $\left(T_{2}\right)$, and on the morning of the first postoperative day $\left(T_{3}\right)$. The comet assay was used to detect basal and oxidative DNA damage. Biomarkers of lipid peroxidation, such as 4-hydroxynonenal (4-HNE) and 8-iso-prostaglandin $\mathrm{F}_{2 \alpha}$ (8-isoprostane), as well as protein oxidation, were assessed by ELISA. Finally, ferric-reducing antioxidant power (FRAP), an assay which measures the total antioxidant capacity, has been determined to assess the reducing ability of the serum samples. The percentage (\%) of DNA strand breaks increased in time $(8.2 \%, 9.5 \%$, and $10.6 \%$ at $T_{1}, T_{2}$, and $T_{3}$, respectively). The $\%$ of oxidized pyrimidines, but not purines, increased in time. Markers of oxidative stress (4-HNE and 8-isoprostane) both increased from $T_{1}$ to $T_{3}$ [33].

Another small study analyzed the effects of desflurane on oxidative stress by measuring lipid peroxidation (LP), glutathione peroxidase (GSH-Px), erythrocyte superoxide dismutase (SOD), and vitamin $\mathrm{E}$ values in blood serum of patients undergoing elective surgery [34]. Blood samples were collected at baseline and 1 and 12 hours following desflurane exposure. LP values significantly differed between baseline and 1 hour postexposure, but not between baseline and 12 hours postexposure. Both GSH-Px and SOD did not differ significantly between the three time points. Vitamin E levels were significantly lower 1 hour postexposure compared 
to baseline but did not differ between baseline and 12 hours postexposure. These findings suggest that desflurane increases oxidative stress as demonstrated by increased LP values and decreased vitamin $\mathrm{E}$ values 1 hour following desflurane exposure [34]. However, the fact that all measured levels returned to baseline values at the $12^{\text {th }}$ hour postexposure casts doubt on the clinical significance of this finding.

The impact of desflurane and sevoflurane on oxidative stress parameters has further been investigated both in mothers and newborns undergoing elective cesarean section [35]. In this randomized study, patients were assigned to either a desflurane (group D) or sevoflurane (group S) group. Blood samples were obtained from mothers at baseline and at the end of surgery, while umbilical artery samples were collected at delivery. Total oxidant status, total antioxidant capacity status, lipid hydroperoxide, and free sulfhydryl levels were measured, and the oxidative stress index was calculated. Preoperative levels did not differ between the two groups. Postoperatively, total oxidant status and oxidative stress index levels were substantially decreased in both groups compared with preoperative levels. Among the two groups, postoperative levels of lipid hydroperoxide, total oxidant status, and oxidative stress index were markedly higher in group $\mathrm{D}(p=0.003, p=0.005$, and $p=0.04$, respectively) compared with group S. Free sulfhydryl levels and total antioxidant capacity status did not differ significantly among groups. This study demonstrated that both agents induce oxidative stress. However, compared with desflurane, sevoflurane demonstrated more favorable effects in terms of oxidative stress [35].

3.3. Isoflurane. Isoflurane was isolated from methyl ethyl ethers in 1965. It shows physical characteristics that are close to the ideal for an anesthetic agent, being highly stable and nonflammable and having a low blood solubility, and it undergoes very little biodegradation. Clinical trials conducted in the 1970's showed that isoflurane leads to a rapid induction and recovery and excellent muscle relaxation and is capable of maintaining blood pressure and cardiac output. It furthermore leads to respiratory depression along with an increase in cerebral blood flow and uterine muscle relaxation [36].

Previous studies have shown that isoflurane may generate the neurotoxicity associated with the pathogenesis of Alzheimer's disease, including accumulation of $\beta$-amyloid protein and phosphorylation of Tau protein [37-39], and cause neurobehavior deficits [40-42]. A recent study analyzed whether isoflurane causes DNA damage [43]. Since oxidative stress, caspase-activated DNase (CAD), and the p53 signaling pathway are all implicated in DNA damage, ROS, CAD, and p53 were measured in $\mathrm{H} 4$ human neuroglioma cells. They were able to demonstrate that isoflurane induces DNA damage by the following mechanisms: (1) isoflurane induced oxidative stress, leading to caspase-3 activation and consequently CAD activation, causing DNA damage, and (2) isoflurane prevented the repair of DNA damage through reduction of p53 levels [43].

A small prospective randomized study compared isoflurane with propofol for maintenance of anesthesia regarding their antioxidant effects on 30 adult patients without comorbidities undergoing elective minor surgery [44]. 15 patients each were randomized to maintenance of anesthesia with either propofol or isoflurane. For the primary outcomes, aqueous plasma oxidizability and total antioxidant performance (TAP) were measured by fluorometry as well as several individual antioxidants by high-performance liquid chromatography. As a secondary outcome, oxidized genetic damage (7,8-dihydro-8-oxoguanine, known as 8-oxo-Gua) was investigated by the comet assay. Maintenance of anesthesia with either propofol or isoflurane resulted in a significant decrease of plasma $\alpha$-tocopherol, but not other antioxidants including uric acid, carotenoids, and retinol. Propofol, but not isoflurane, significantly increased antiinflammatory/antioxidant plasma $\gamma$-tocopherol concentration in patients $(p<0.001)$. Both anesthesia types equally enhanced hydrophilic antioxidant capacity and TAP. Furthermore, 8-oxo-Gua remained unchanged during anesthesia in both groups, thus showing that neither of the two anesthetics leads to oxidative DNA damage in this patient group. Thus, the authors concluded that maintenance of anesthesia with either propofol or isoflurane increases both hydrophilic and total antioxidant capacity in plasma, but only propofol anesthesia increases plasma $\gamma$-tocopherol concentration [44].

3.4. Nitrous Oxide. Nitrous oxide $\left(\mathrm{N}_{2} \mathrm{O}\right)$ was first used in the late $18^{\text {th }}$ century and has gained wide popularity in the anesthetic world because of its fast uptake and elimination, in addition to its analgesic effects [45]. $\mathrm{N}_{2} \mathrm{O}$ is the least potent among the currently available inhalation agents. It seems to act on multiple targets, including noncompetitive inhibition of N-Methyl-D-Aspartate (NMDA) and non-NMDA glutamate receptors, nicotinic acetylcholine $(\mathrm{nACH})$ receptors, and gamma-aminobutyric acid (GABA) receptors, along with activity on calcium and potassium channels $[46,47]$. The analgesic effects of $\mathrm{N}_{2} \mathrm{O}$ is caused by the inhibition of supraspinal NMDA receptors [48]. The low toxicity of modern anesthetic agents and increasing evidence of the deleterious effects of $\mathrm{N}_{2} \mathrm{O}$ have led to a steady decline in the use of this agent in general anesthesia $[49,50]$. Various types of cytogenetic damage, including increased sister chromatid exchange [51], chromosomal aberrations [52], and DNA single-strand breaks [53], have been observed in operating room staff exposed to $\mathrm{N}_{2} \mathrm{O}$. A study compared nurses who had been exposed to $\mathrm{N}_{2} \mathrm{O}$ to a group of volunteer nurses not being exposed to the agent [54]. The numbers of binucleated cells carrying between one to four micronuclei were recorded individually, and the means of the total cytokinesis-block micronucleus assay (CBMNt) and total ARA-enhanced micronucleus assay (CBMNAt) frequencies were compared between the exposed and control populations. The CBMN assay measures DNA damage, cytostasis, and cytotoxicity in different tissue types, including lymphocytes [55]. The mean frequency of CBMNt in lymphocytes of nurses with $\mathrm{N}_{2} \mathrm{O}$ exposure was significantly increased over the controls. A linear correlation between duration of exposure and the CBMNt or CBMNAt frequencies showed that cytogenetic damage might be associated with chronic 
ambient $\mathrm{N}_{2} \mathrm{O}$ exposure. In conclusion, this study showed that chronic $\mathrm{N}_{2} \mathrm{O}$ exposure may lead to some trans-acting DNA damage or increased error-prone DNA repair in in vitro cultivated lymphocytes. These lesions would appear in vitro as micronuclei or some other cytogenetic damage [54].

\section{Intravenous Anesthetics}

Intravenous anesthesia can be traced back to the mid- $17^{\text {th }}$ century, where it has been used both in animals and humans and entered the era of modern anesthesia with the release of thiopental in 1936 [56]. Since then, the pharmacodynamics and pharmacokinetics of intravenous anesthetics have been studied in much greater detail. Building on this knowledge and the availability of short-acting drugs has shifted the attention of the anesthesiologist to the administration of anesthesia not based on the needs of the population but on the individual needs of the patient.

We will subsequently analyze the most common intravenous anesthetics regarding their potential for inducing or mitigating oxidative stress.

Figure 2 represents the chemical structures of the intravenous anesthetics discussed in this paper.

Table 2 summarizes the intravenous anesthetic agents discussed in this paper regarding their effect on oxidative stress.

4.1. Propofol. Propofol belongs to a group of alkylphenols which have all in common that they are particularly lipid soluble and insoluble in an aqueous solution. It acts mainly by enhancing GABA-induced chloride current through its binding to the $\beta$-subunit of $\mathrm{GABA}_{\mathrm{A}}$ receptor $[57,58]$. Furthermore, acetylcholine release in the hippocampus and prefrontal cortex is diminished. The $\alpha_{2}$-adrenoreceptor system also appears to be involved in mediating the sedative effects of propofol [59].

Propofol has been shown to possess antioxidant properties, and a reduction of oxidative stress has been associated with an improved postoperative outcome, thus making it an ideal anesthetic in clinical practice $[10,60,61]$. Indeed, a study took it upon itself to analyze whether propofol exerts neuroprotective effects [62]. Human SH-SY5Y cells were pretreated with ferric ammonium citrate (FAC) and then treated with propofol. FAC pretreatment induced cell damage. When these cells were treated with propofol $(5 \mu \mathrm{M})$ for 6 hours, increased cell viability could be observed. To assess whether this effect correlated with iron levels, the cellular iron levels were additionally tested. Propofol decreased the cellular iron concentration dramatically, indicating that propofol suppresses the iron increase induced by FAC. Additionally, the authors assessed the ROS and malondialdehyde (MDA) levels of SHSY5Y cells. The results showed that FAC significantly increased ROS and MDA levels. Propofol significantly decreased ROS levels but did not significantly decrease MDA levels. Further, the authors noted that the FAC-induced increase in ROS was closely associated with a decrease in the mitochondrial membrane potential (MMP), which constitutes an essential component in the process of energy storage during oxidative phosphorylation [63]. Propofol was able to suppress the decrease of MMP induced by FAC. FAC induced increased cellular apoptosis, which could be significantly inhibited by treatment with propofol. Treatment with FAC leads to an increase in the expression of inflammatory markers, such as interleukin(IL-) 6 and cyclooxygenase 2. Propofol significantly inhibited the expression of both proteins, indicating that propofol might influence the inflammatory pathway. In summary, this study demonstrated that propofol influences both levels of oxidative stress and inflammation [62]. The antioxidative properties of propofol were also observed in human cardiac cells, and the protective effect was attributed to an activation of the JNK signaling pathway [64].

A prospective, randomized trial by Guo et al. [65] evaluated in 60 patients undergoing aneurysm clipping the role of propofol postconditioning on oxidative stress and postoperative cognitive function. The patients were randomized into a propofol postconditioning group or a sevoflurane group. In both groups, sevoflurane $(0.5 \%-2 \%)$ was used for maintenance of anesthesia. In the propofol postconditioning group, the concentration of sevoflurane was diminished following temporary clip removal, and propofol was subsequently started. Blood samples were taken at 6 time points: before induction, immediately after clip removal, at the end of the operation, 24 hours postsurgery, and 3 and 7 days postsurgery. Oxidative stress (hydroxyl radicals $(\mathrm{OH}), 8$-isoprostane, $\alpha$-tocopherol, $\gamma$-tocopherol, and SOD) and cognitive function were measured. Between the end of the operation to 7 days postsurgery, both $\mathrm{OH}$ and 8-isoprostane levels were markedly lower in the propofol group, while levels of $\gamma$-tocopherol and SOD, markers of antioxidant capacity, increased significantly in the propofol group. To assess DNA damage, a cytokinesis-block micronucleus test was performed. Postoperatively, micronuclei as well as nucleoplasmic bridges were seen more frequently in the sevoflurane group. Finally, the propofol postconditioning group had markedly higher scores on cognitive function tests compared with the sevoflurane group after the surgery [65].

4.2. Ketamine. Ketamine, an arylcyclohexylamine related to phencyclidine, was developed in the 1960's and was originally produced as a racemic mixture of the $\mathrm{R}$ and $\mathrm{S}$ enantiomers. In some European countries, a single enantiomer formulation $(\mathrm{S}(+))$ is available which is more potent in most clinical and experimental settings. Ketamine has been described to cause a sense of disconnection from the environment, leading to the term "dissociative anesthesia" [66]. The key site(s) of ketamine action have not been entirely elucidated; however, ketamine has been identified as an antagonist at the NMDA receptor in the brain and spinal cord [67]. The role of NMDA receptors in mediating analgesia is supported by experimental data, while their role in inducing anesthesia is unclear [68]. At clinically relevant concentrations, ketamine also inhibits nAch receptors, which could contribute to analgesia but do not seem to affect its sedative properties [69]. Furthermore, ketamine influences also $\mathrm{Na}^{+}$channels and binds to $\mu$ - and $\kappa$-opioid receptors, thus possessing some local anesthetic properties [70]. However, the minimal effect of naloxone on ketamine actions suggests a limited role for these receptors. Ketamine has 


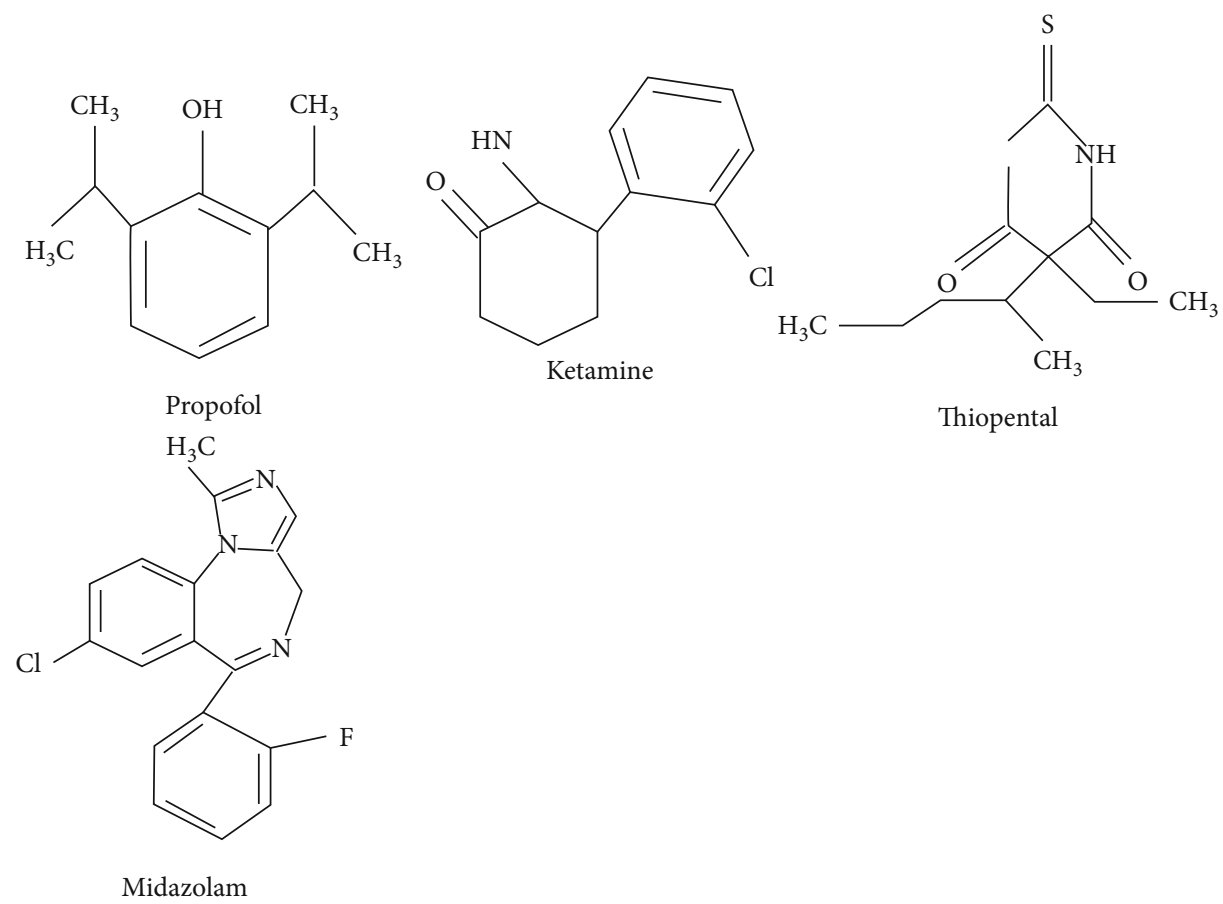

Figure 2: Chemical structures of the intravenous anesthetics discussed in this paper.

TABLE 2: Intravenous anesthetic agents investigated regarding their effect on oxidative stress.

\begin{tabular}{lccc}
\hline Study reference & Anesthetic agent & Studied population & Main effect on oxidative stress \\
\hline$[62]$ & Propofol & Human study & $\uparrow$ Cell viability, MMP, $\downarrow$ ROS, IL-6, and COX2 levels \\
{$[64]$} & Propofol & Human study & Activation of the JNK signaling pathway \\
{$[65]$} & Propofol, sevoflurane & Human study & $\downarrow$ OH, 8-isoprostane, micronuclei, nucleoplasmic bridges, $\uparrow \gamma$-tocopherol, \\
{$[72]$} & Propofol, ketamine & Human study & $\downarrow$ SOD compared with sevoflurane \\
{$[82]$} & Propofol, midazolam & Human study & $\downarrow$ IL-6, IL-8, MDA and $\uparrow$ SOD levels compared with midazolam \\
\hline
\end{tabular}

COX: cyclooxygenase; GSH-Px: glutathione peroxidase; IL: interleukin; JNK: c-Jun N-terminal kinase; LP: lipid peroxidation; MDA: malondialdehyde; MMP: mitochondrial membrane potential; 'OH: hydroxyl radical; ROS: reactive oxygen species; SOD: superoxide dismutase; TAC: total antioxidant capacity; TTM: total thiol molecules.

unique properties combining depressed consciousness with an increased sympathetic tone, thus making it a useful induction agent in patients with hypovolemia [71].

The antioxidant properties of ketamine have been studied in a head-to-head comparison with propofol in 80 patients undergoing general surgeries [72]. 40 patients each were randomized either in the ketamine group, where general anesthesia was induced by intravenous injection of ketamine, and a propofol group, where propofol constituted the induction agent. Blood samples were obtained 15 minutes after induction, and the following oxidative markers were assessed: serum SOD activity, GSH-Px, CAT and levels of LP, total antioxidant capacity (TAC), and total thiol molecules (TTM). This study demonstrated that propofol significantly increased TTM and TAC ( $p=0.001$ and $p=0.003$, respectively) and decreased LP $(p=0.014), \operatorname{SOD}(p=0.047)$, and GSH-Px $(p=0.037)$ activity compared to the ketamine group. The level of CAT did not differ significantly between the two groups. Thus, pro- pofol possesses greater antioxidant capacities compared with ketamine [72].

4.3. Thiopental. Barbiturates were discovered in the early $20^{\text {th }}$ century, with hexobarbital being the first agent of this class. In 1934, thiopental became preferred clinically due to its rapid onset of action and short duration. Apart from the action on the $\mathrm{GABA}_{\mathrm{A}}$ receptor, the mechanism of action of barbiturates is largely unknown. NMDA receptors might also play a role in the effects of barbiturates [73]. Barbiturates enhance the synaptic actions of inhibitory neurotransmitters and block the synaptic actions of excitatory neurotransmitters in the central nervous system (CNS) [74]. GABA is the main inhibitory neurotransmitter in the $\mathrm{CNS}$, and the $\mathrm{GABA}_{\mathrm{A}}$ receptor is the only site proven to be involved in barbiturate-induced anesthesia [75]. The inhibition of synaptic actions of excitatory neurotransmitters involves mainly glutamate and acetylcholine. Thiopental may exert GABA-independent effects on the 
glutaminergic-NMDA system. Two studies demonstrated that thiopental decreases extracellular glutamate levels in the CNS and decreases NMDA-gated currents in a concentrationdependent manner $[73,76]$.

To date, no studies exist which analyzed the antioxidant effects of thiopental on humans. However, animal and in vitro studies exist which suggest that thiopental possesses antioxidant capacities, even though the effect has been observed to be smaller than that of propofol [77].

4.4. Midazolam. The benzodiazepines are a class of drugs often used in anesthesia as anxiolytics, sedatives, and hypnotics. In clinical practice, midazolam is frequently used immediately before induction of anesthesia. Like most intravenous anesthetics, benzodiazepines exert their action through $\mathrm{GABA}_{\mathrm{A}}$ receptors [78]. They are widely prescribed, and addiction to these drugs is a worldwide concern. The $\alpha_{2}-$ and $\alpha_{3}$-subunits of the $\mathrm{GABA}_{\mathrm{A}}$ receptors are implicated as key mediators of the reward-related effects of benzodiazepines [79]. All benzodiazepines have hypnotic, sedative, anxiolytic, amnesic, anticonvulsant, and centrally produced muscle-relaxing properties. The mechanism of action of benzodiazepines is reasonably well understood [80]. The $\alpha$ subunit of the GABA receptor occurs in six isoforms $\left(\alpha_{1}\right.$ $\alpha_{6}$ ) [81]. Sedation, anterograde amnesia, and anticonvulsant properties are mediated via $\alpha_{1}$-subunits [80], and anxiolysis and muscle relaxation are mediated via the $\alpha_{2}$-subunits.

The effects of midazolam on inflammation and oxidative stress have been compared with propofol in children with congenital heart disease undergoing cardiac surgery [82]. 32 children were randomly assigned to a midazolam or propofol (both combined with low dose fentanyl) group. Blood samples were collected preoperatively $\left(T_{0}\right)$, at $2 \mathrm{~h}$ following the release of the aorta cross-clamp $\left(T_{1}\right)$, and at $24 \mathrm{~h}$ after operation $\left(T_{2}\right)$, and the following oxidative stress markers are measured: IL-6, IL-8, SOD, and MDA levels. After cardiopulmonary bypass, IL-6 and IL- 8 levels were markedly increased in both groups at $T_{1}$ and $T_{2}$ compared with $T_{0}$. However, the propofol group exhibited lower IL-6 and IL-8 levels at $T_{1}$ and $T_{2}$ compared with the midazolam group. Similarly, MDA levels were significantly increased, while SOD levels were significantly decreased at $T_{1}$ and $T_{2}$ in both groups. The propofol group exhibited lower MDA levels and higher SOD levels compared with the midazolam group. Thus, this study showed that propofol exerts greater potential to decrease inflammation and oxidative stress compared with midazolam.

\section{Conclusions}

Surgical interventions have been associated with increased levels of oxidative stress, and the increase has been closely correlated with the extent of the surgical procedure. Furthermore, increased levels of oxidative stress have been associated with worse clinical outcomes and increased postoperative complications. Given the magnitude of anesthesia being delivered each day, procedural safety and few postoperative complications should be a top priority for every anesthesiologist. The anesthesiologist's armamentarium has broadened significantly over the past decades, and several induction methods using different anesthetic agents are at the disposal of the anesthesiologist. However, as highlighted in this review, some anesthetic agents have a greater potential to induce oxidative stress as compared with others, with some agents even exerting antioxidative effects. Among the most commonly used induction agents, propofol seems to possess the greatest benefits in terms of reducing oxidative stress. Since propofol constitutes the most widely used induction agent in the developed world, the antioxidative properties it possesses certainly come in handy.

Given the impact that anesthesiologists can have on the patients' well-being, we should definitely care about anesthesia-induced oxidative stress.

\section{Abbreviations}

8-OHdG: 8-Hydroxy-2' -deoxyguanosine

ADMA: Asymmetric dimethylarginine

CABG: Coronary artery bypass grafting

CNS: Central nervous system

d-ROMs: Derivatives of reactive oxygen metabolites

GABA: Gamma-aminobutyric acid

LP: $\quad$ Lipid peroxidation

MDA: Malondialdehyde

MMP: Mitochondrial membrane potential

$\mathrm{N}_{2} \mathrm{O}: \quad$ Nitrous oxide

nACH: Nicotinic acetylcholine

NADPH: Nicotinamide adenine dinucleotide phosphate

NMDA: N-Methyl-D-Aspartate

NOX2: Nicotinamide adenine dinucleotide phosphate oxidase isoform 2

ROS: $\quad$ Reactive oxygen species

RNS: $\quad$ Reactive nitrogen species

SOD: $\quad$ Superoxide dismutase.

\section{Conflicts of Interest}

The authors declare that there is no conflict of interest.

\section{Authors' Contributions}

T.S. substantially contributed to the conception and design of the work and the acquisition, analysis, and interpretation of data; T.S. drafted the manuscript; T.S., C.V.S., G.L., and H.T. revised it critically for important intellectual content; and T.S., C.V.S., G.L., and H.T. approved the final version of the review article.

\section{References}

[1] S. P. Hussain, L. J. Hofseth, and C. C. Harris, "Radical causes of cancer," Nature Reviews Cancer, vol. 3, no. 4, pp. 276-285, 2003.

[2] L. H. Sanders and J. T. Greenamyren, "Oxidative damage to macromolecules in human Parkinson disease and the rotenone model," Free Radical Biology and Medicine, vol. 62, pp. 111120, 2013. 
[3] B. C. Song, N. S. Joo, G. Aldini, and K. J. Yeum, "Biological functions of histidine-dipeptides and metabolic syndrome," Nutrition Research and Practice, vol. 8, no. 1, pp. 3-10, 2014.

[4] T. Senoner and W. Dichtl, "Oxidative stress in cardiovascular diseases: still a therapeutic target?," Nutrients, vol. 11, no. 9, p. 2090, 2019.

[5] S. I. Liochev, "Reactive oxygen species and the free radical theory of aging," Free Radical Biology \& Medicine, vol. 60, pp. 1-4, 2013.

[6] K. M. Holmström and T. Finkel, "Cellular mechanisms and physiological consequences of redox-dependent signalling," Nature Reviews. Molecular Cell Biology, vol. 15, no. 6, pp. 411-421, 2014.

[7] T. Finkel, "Signal transduction by reactive oxygen species," The Journal of Cell Biology, vol. 194, no. 1, pp. 7-15, 2011.

[8] R. S. Balaban, S. Nemoto, and T. Finkel, "Mitochondria, oxidants, and aging," Cell, vol. 120, no. 4, pp. 483-495, 2005.

[9] E. Omling, A. Jarnheimer, J. Rose, J. Björk, J. G. Meara, and L. Hagander, "Population-based incidence rate of inpatient and outpatient surgical procedures in a high-income country," The British Journal of Surgery, vol. 105, no. 1, pp. 86-95, 2018.

[10] T. Senoner, S. Schindler, S. Stättner, D. Öfner, J. Troppmair, and F. Primavesi, "Associations of oxidative stress and postoperative outcome in liver surgery with an outlook to future potential therapeutic options," Oxidative Medicine and Cellular Longevity, vol. 2019, 18 pages, 2019.

[11] R. Arsalani-Zadeh, S. Ullah, S. Khan, and J. MacFie, "Oxidative Stress in Laparoscopic_Versus_Open Abdominal Surgery: A Systematic Review," Journal of Surgical Research, vol. 169, no. 1, pp. e59-e68, 2011

[12] M. Tsuchiya, K. Shiomoto, K. Mizutani et al., "Reduction of oxidative stress a key for enhanced postoperative recovery with fewer complications in esophageal surgery patients," Medicine, vol. 97, no. 47, p. e12845, 2018.

[13] D. Plicner, P. Mazur, J. Sadowski, and A. Undas, "Asymmetric dimethylarginine and oxidative stress following coronary artery bypass grafting: associations with postoperative outcome," European Journal of Cardio-Thoracic Surgery, vol. 45, no. 5, pp. e136-e141, 2014.

[14] J.-C. Hsu, C.-Y. Huang, S.-L. Chuang et al., "Long term outcome of postoperative atrial fibrillation after cardiac surgerya propensity score-matched cohort analysis," Frontiers in Cardiovascular Medicine, vol. 8, 2021.

[15] J. H. Y. Wu, R. Marchioli, M. G. Silletta et al., "Oxidative stress biomarkers and incidence of postoperative atrial fibrillation in the omega-3 fatty acids for prevention of postoperative atrial fibrillation (OPERA) trial," Journal of the American Heart Association, vol. 4, no. 5, 2015.

[16] F. X. Whalen, D. R. Bacon, and H. M. Smith, "Inhaled anesthetics: an historical overview," Best Practice and Research: Clinical Anaesthesiology, vol. 19, no. 3, pp. 323-330, 2005.

[17] E. I. Eger, D. E. Raines, S. L. Shafer, H. C. Hemmings, and J. M. Sonner, "Is a new paradigm needed to explain how inhaled anesthetics produce immobility?," Anesthesia and Analgesia, vol. 107, no. 3, pp. 832-848, 2008.

[18] H. C. Hemmings, M. H. Akabas, P. A. Goldstein, J. R. Trudell, B. A. Orser, and N. L. Harrison, "Emerging molecular mechanisms of general anesthetic action," Trends in Pharmacological Sciences, vol. 26, no. 10, pp. 503-510, 2005.

[19] J. M. Sonner, J. F. Antognini, R. C. Dutton et al., "Inhaled anesthetics and immobility: mechanisms, mysteries, and minimum alveolar anesthetic concentration," Anesthesia and Analgesia, vol. 97, no. 3, pp. 718-740, 2003.

[20] M. Juhász, L. Molnár, B. Fülesdi, T. Végh, D. Páll, and C. Molnár, "Effect of sevoflurane on systemic and cerebral circulation, cerebral autoregulation and $\mathrm{CO}_{2}$ reactivity," $B M C$ Anesthesiology, vol. 19, no. 1, p. 109, 2019.

[21] A. Holmström and J. Åkeson, "Sevoflurane induces less cerebral vasodilation than isoflurane at the same A-line ${ }^{\circledR}$ autoregressive index level," Acta Anaesthesiologica Scandinavica, vol. 49, no. 1, pp. 16-22, 2005.

[22] Y. Qin, J. Ni, L. Kang, Z. Zhong, L. Wang, and S. Yin, "Sevoflurane effect on cognitive function and the expression of oxidative stress response proteins in elderly patients undergoing radical surgery for lung cancer," Journal of the College of Physicians and Surgeons Pakistan, vol. 29, no. 1, pp. 12-15, 2019.

[23] M. Herrmann, P. Vos, M. T. Wunderlich, C. H. M. M. De Bruijn, and K. J. B. Lamers, "Release of glial tissue-specific proteins after acute stroke: a comparative analysis of serum concentrations of protein S-100B and glial fibrillary acidic protein," Stroke, vol. 31, no. 11, pp. 2670-2677, 2000.

[24] Z. Cukurova, H. Cetingok, S. Ozturk et al., "DNA damage effects of inhalation anesthetics in human bronchoalveolar cells," Medicine, vol. 98, no. 32, p. e16518, 2019.

[25] A. Valavanidis, T. Vlachogianni, and C. Fiotakis, "8-Hydroxy2' -deoxyguanosine (8-OHdG): a critical biomarker of oxidative stress and carcinogenesis," Journal of Environmental Science and Health, Part C, vol. 27, no. 2, pp. 120-139, 2009.

[26] V. Sanchez, S. . D. Feinstein, N. Lunardi et al., "General anesthesia causes long-term impairment of mitochondrial morphogenesis and synaptic transmission in developing rat brain," Anesthesiology, vol. 115, no. 5, pp. 992-1002, 2011.

[27] V. Jevtovic-Todorovic, R. E. Hartman, Y. Izumi et al., "Early exposure to common anesthetic agents causes widespread neurodegeneration in the developing rat brain and persistent learning deficits," The Journal of Neuroscience, vol. 23, no. 3, pp. 876-882, 2003.

[28] V. Jevtovic-Todorovic, A. R. Absalom, K. Blomgren et al., "Anaesthetic neurotoxicity and neuroplasticity: an expert group report and statement based on the BJA Salzburg Seminar," British Journal of Anaesthesia, vol. 111, no. 2, pp. 143151, 2013.

[29] A. J. Davidson, N. Disma, J. C. de Graaff et al., "Neurodevelopmental outcome at 2 years of age after general anaesthesia and awake-regional anaesthesia in infancy (GAS): an international multicentre, randomised controlled trial," Lancet, vol. 387, no. 10015, pp. 239-250, 2016.

[30] T. J. Ebert, F. Perez, T. D. Uhrich, and M. A. Deshur, "Desflurane-mediated sympathetic activation occurs in humans despite preventing hypotension and baroreceptor unloading," Anesthesiology, vol. 88, no. 5, pp. 1227-1232, 1998.

[31] G. Cinnella, G. Vendemiale, M. Dambrosio et al., "Effect of propofol, sevoflurane and desflurane on systemic redox balance," International Journal of Immunopathology and Pharmacology, vol. 20, no. 3, pp. 585-593, 2007.

[32] F. R. Nogueira, L. G. Braz, L. R. de Andrade et al., "Evaluation of genotoxicity of general anesthesia maintained with desflurane in patients under minor surgery," Environmental and Molecular Mutagenesis, vol. 57, no. 4, pp. 312-316, 2016.

[33] F. R. Nogueira, L. G. Braz, K. M. Souza et al., "Comparison of DNA damage and oxidative stress in patients anesthetized with desflurane associated or not with nitrous oxide: a 
prospective randomized clinical trial," Anesthesia and Analgesia, vol. 126, no. 4, pp. 1198-1205, 2018.

[34] F. Eroglu, L. Yavuz, B. G. Ceylan et al., "New volatile anesthetic, desflurane, reduces vitamin e level in blood of operative patients via oxidative stress," Cell Biochemistry and Function, vol. 28, no. 3, pp. 211-216, 2010.

[35] S. Yalcin, H. Aydoğan, H. H. Yuce et al., "Effects of sevoflurane and desflurane on oxidative stress during general anesthesia for elective cesarean section," Wiener Klinische Wochenschrift, vol. 125, no. 15-16, pp. 467-473, 2013.

[36] C. Ball and R. N. Westhorpe, "Isoflurane," Anaesthesia and Intensive Care, vol. 35, no. 4, p. 467, 2007.

[37] H. Wei, B. Kang, W. Wei et al., "Isoflurane and sevoflurane affect cell survival and BCL-2/BAX ratio differently," Brain Research, vol. 1037, no. 1-2, pp. 139-147, 2005.

[38] Z. Xie, Y. Dong, U. Maeda et al., "The common inhalation anesthetic isoflurane induces apoptosis and increases amyloid $\beta$ protein levels," Anesthesiology, vol. 104, no. 5, pp. 988-994, 2006.

[39] R. G. Eckenhoff, J. S. Johansson, H. Wei et al., "Inhaled anesthetic enhancement of amyloid- $\beta$ oligomerization and cytotoxicity," Anesthesiology, vol. 101, no. 3, pp. 703-709, 2004.

[40] J. K. Callaway, N. C. Jones, and C. F. Royse, "Isoflurane induces cognitive deficits in the Morris water maze task in rats," European Journal of Anaesthesiology, vol. 29, no. 5, pp. 239-245, 2012.

[41] D. J. Culley, M. Baxter, R. Yukhananov, and G. Crosby, “The memory effects of general anesthesia persist for weeks in young and aged rats," Anesthesia and Analgesia, vol. 96, no. 4, pp. 1004-1009, 2003.

[42] S. L. Bianchi, T. Tran, C. L. Liu et al., "Brain and behavior changes in 12-month-old Tg2576 and nontransgenic mice exposed to anesthetics," Neurobiology of Aging, vol. 29, no. 7, pp. 1002-1010, 2008.

[43] C. Ni, C. Li, Y. Dong, X. Guo, Y. Zhang, and Z. Xie, “Anesthetic isoflurane induces DNA damage through oxidative stress and p53 pathway," Molecular Neurobiology, vol. 54, no. 5, pp. 3591-3605, 2017.

[44] M. G. Braz, L. G. Braz, C. M. M. Freire et al., "Isoflurane and propofol contribute to increasing the antioxidant status of patients during minor elective surgery: a randomized clinical study," Medicine (Baltimore), vol. 94, no. 31, p. e1266, 2015.

[45] V. Lew, E. McKay, and M. Maze, "Past, present, and future of nitrous oxide," British Medical Bulletin, vol. 125, no. 1, pp. 103-119, 2018.

[46] T. Yamakura and R. A. Harris, "Effects of gaseous anesthetics nitrous oxide and xenon on ligand-gated ion channels: comparison with isoflurane and ethanol," Anesthesiology, vol. 93, no. 4, pp. 1095-1101, 2000.

[47] J. Hendrickx, P. Peyton, R. Carette, and A. De Wolf, "Inhaled anaesthetics and nitrous oxide," European Journal of Anaesthesiology, vol. 33, no. 9, pp. 611-619, 2016.

[48] S. Sawamura, M. Obara, K. Takeda, M. Maze, and K. Hanaoka, "Corticotropin-releasing factor mediates the antinociceptive action of nitrous oxide in rats," Anesthesiology, vol. 99, no. 3, pp. 708-715, 2003.

[49] S. G. D. Hert, "The current place of nitrous oxide in clinical practice," European Journal of Anaesthesiology, vol. 32, no. 8, pp. 517-520, 2015.

[50] P. S. Myles, K. Leslie, M. T. V. Chan et al., "Avoidance of nitrous oxide for patients undergoing major surgery: a ran- domized controlled trial," Anesthesiology, vol. 107, no. 2, pp. 221-231, 2007.

[51] S. Şardaş, H. Cuhruk, A. E. Karakaya, and Y. Atakurt, "Sisterchromatid exchanges in operating room personnel," Mutation Research/Genetic Toxicology, vol. 279, no. 2, pp. 117-120, 1992.

[52] J. Karelová, A. Jablonická, J. Gavora, and Ł. Hanoi, "Chromosome and sister-chromatid exchange analysis in peripheral lymphocytes, and mutagenicity of urine in anesthesiology personnel," International Archives of Occupational and Environmental Health, vol. 64, no. 4, pp. 303-306, 1992.

[53] M. Reitz, R. Coen, and E. Lanz, "DNA single-strand breaks in peripheral lymphocytes of clinical personnel with occupational exposure to volatile inhalational anesthetics," Environmental Research, vol. 65, no. 1, pp. 12-21, 1994.

[54] W. P. Chang, S. R. Lee, J. Tu, and S. S. Hseu, "Increased micronucleus formation in nurses with occupational nitrous oxide exposure in operating theaters," Environmental and Molecular Mutagenesis, vol. 27, no. 2, pp. 93-97, 1996.

[55] P. Thomas and M. Fenech, "Cytokinesis-block micronucleus cytome assay in lymphocytes," Methods in Molecular Biology, vol. 682, pp. 217-234, 2011.

[56] R. Jarman, "History of intravenous anaesthesia with ten years' experience in the use of pentothal sodium," Postgraduate Medical Journal, vol. 22, no. 252, pp. 311-318, 1946.

[57] D. Ruesch, E. Neumann, H. Wulf, and S. A. Forman, “An allosteric coagonist model for propofol effects on $\alpha 1 \beta 2 \gamma 2 \mathrm{~L} \gamma$-aminobutyric acid type A receptors," Anesthesiology, vol. 116, no. 1, pp. 47-55, 2012.

[58] M. D. Krasowski, V. V. Koltchine, C. E. Rick, Q. Ye, S. E. Finn, and N. L. Harrison, "Propofol and other intravenous anesthetics have sites of action on the $\Gamma$-aminobutyric acid type A receptor distinct from that for isoflurane," Molecular Pharmacology, vol. 53, no. 3, pp. 530-538, 1998.

[59] T. Kushikata, K. Hirota, H. Yoshida, T. Kubota, H. Ishihara, and A. Matsuki, "Alpha-2 adrenoceptor activity affects propofol-induced sleep time," Anesthesia and Analgesia, vol. 94, no. 5, pp. 1201-1206, 2002.

[60] M. Y. Kang, M. Tsuchiya, L. Packer, and M. Manabe, "In vitro study on antioxidant potential of various drugs used in the perioperative period," Acta Anaesthesiologica Scandinavica, vol. 42, no. 1, pp. 4-12, 1998.

[61] M. Tsuchiya, A. Asada, E. Kasahara, E. F. Sato, M. Shindo, and M. Inoue, "Antioxidant protection of propofol and its recycling in erythrocyte membranes," American Journal of Respiratory and Critical Care Medicine, vol. 165, no. 1, pp. 54-60, 2002.

[62] Y. Zhang, Y. Zuo, B. Li et al., "Propofol prevents oxidative stress and apoptosis by regulating iron homeostasis and targeting JAK/STAT3 signaling in SH-SY5Y cells," Brain Research Bulletin, vol. 153, pp. 191-201, 2019.

[63] L. D. Zorova, V. A. Popkov, E. Y. Plotnikov et al., "Mitochondrial membrane potential," Analytical Biochemistry, vol. 552, pp. 50-59, 2018.

[64] L. Han, Q. Zhuo, Y. Zhou, and Y. Qian, "Propofol protects human cardiac cells against chemical hypoxiainduced injury by regulating the JNK signaling pathways," Experimental and Therapeutic Medicine, vol. 19, no. 3, 2020.

[65] Y. L. Di Guo, H. Wang, X. Wang et al., "Propofol postconditioning after temporary clipping reverses oxidative stress in aneurysm surgery," The International Journal of Neuroscience, vol. 129, no. 2, pp. 155-164, 2019. 
[66] E. F. Domino, "Taming the ketamine tiger," Anesthesiology, vol. 113, no. 3, pp. 678-684, 2010.

[67] N. A. Anis, S. C. Berry, N. R. Burton, and D. Lodge, "The dissociative anaesthetics, ketamine and phencyclidine, selectively reduce excitation of central mammalian neurones by N-methyl-aspartate," British Journal of Pharmacology, vol. 79, no. 2, pp. 565-575, 1983.

[68] S. M. South, "A conditional deletion of the NR1 subunit of the NMDA receptor in adult spinal cord dorsal horn reduces NMDA currents and injury-induced pain," The Journal of Neuroscience, vol. 23, no. 12, pp. 5031-5040, 2003.

[69] J. O. Udesky, N. Z. Spence, R. Achiel, C. Lee, and P. Flood, "The role of nicotinic inhibition in ketamine-induced behavior," Anesthesia and Analgesia, vol. 101, no. 2, pp. 407-411, 2005.

[70] K. Hirota, H. Okawa, B. L. Appadu, D. K. Grandy, L. A. Devi, and D. G. Lambert, "Stereoselective interaction of ketamine with recombinant [micro sign], [small kappa, Greek], and [small delta, Greek] opioid receptors expressed in Chinese hamster ovary cells," Anesthesiology, vol. 90, no. 1, pp. 174182, 1999.

[71] H. P. M. M. Gelissen, A. H. Epema, R. H. Henning, H. J. Krijnen, P. J. Hennis, and A. Den Hertog, "Inotropic effects of propofol, thiopental, midazolam, etomidate, and ketamine on isolated human atrial muscle," Anesthesiology, vol. 84, no. 2, pp. 397403., 1996.

[72] E. Khoshraftar, A. Ranjbar, B. Kharkhane, S. Heidary, Z. Gharebaghi, and N. Zadkhosh, "Antioxidative effects of propofol vs. ketamin in individuals undergoing surgery," Archives of Iranian Medicine, vol. 17, no. 7, pp. 468-489, 2014.

[73] H. Liu, T. Dai, and S. Yao, "Effect of thiopental sodium on Nmethyl-D-aspartate-gated currents," Canadian Journal of Anesthesia, vol. 53, no. 5, pp. 442-448, 2006.

[74] S. E. Judge, "Effect of general anaesthetics on synaptic ion channels," British Journal of Anaesthesia, vol. 55, no. 3, pp. 191-200, 1983.

[75] S. L. Tomlin, A. Jenkins, W. R. Lieb, and N. P. Franks, "Preparation of barbiturate optical isomers and their effects on GABA(A) receptors," Anesthesiology, vol. 90, no. 6, pp. 17141722., 1999.

[76] H. Liu and S. Yao, "Thiopental sodium reduces glutamate extracellular levels in rat intact prefrontal cortex," Experimental Brain Research, vol. 167, no. 4, pp. 666-669, 2005.

[77] J. X. Wilson and A. W. Gelb, "Free radicals, antioxidants, and neurologic injury: possible relationship to cerebral protection by anesthetics," Journal of Neurosurgical Anesthesiology, vol. 14, no. 1, pp. 66-79, 2002.

[78] R. Winsky-Sommerer, "Role of GABAAreceptors in the physiology and pharmacology of sleep," European Journal of Neuroscience, vol. 29, no. 9, pp. 1779-1794, 2009.

[79] L. M. Reynolds, E. Engin, G. Tantillo et al., "Differential Roles of $\mathrm{GABA}_{\mathrm{A}}$ Receptor Subtypes in Benzodiazepine-Induced Enhancement of Brain-Stimulation Reward," Neuropsychopharmacology, vol. 37, no. 11, pp. 2531-2540, 2012.

[80] H. Möhler, J. M. Fritschy, and U. Rudolph, "A new benzodiazepine pharmacology," Journal of Pharmacology and Experimental Therapeutics, vol. 300, no. 1, pp. 2-8, 2002.
[81] T. I. Saari, M. Uusi-Oukari, J. Ahonen, and K. T. Olkkola, "Enhancement of GABAergic activity: neuropharmacological effects of benzodiazepines and therapeutic use in anesthesiology," Pharmacological Reviews, vol. 63, no. 1, pp. 243-267, 2011.

[82] W. F. Xia, Y. Liu, Q. S. Zhou, Q. Z. Tang, and H. D. Zou, "Comparison of the effects of propofol and midazolam on inflammation and oxidase stress in children with congenital heart disease undergoing cardiac surgery," Yonsei Medical Journal, vol. 52, no. 2, pp. 326-332, 2011. 